\title{
ESQUIZOFRENIA: UMA REVISÃO
}

\author{
Regina Cláudia Barbosa da Silva ${ }^{\mathbf{1}}$ \\ Universidade Federal de São Paulo - UNIFESP
}

\begin{abstract}
A definição atual de esquizofrenia indica uma psicose crônica idiopática, aparentando ser um conjunto de diferentes doenças com sintomas que se assemelham e se sobrepõem. A esquizofrenia é de origem multifatorial onde os fatores genéticos e ambientais parecem estar associados a um aumento no risco de desenvolver a doença. Esse artigo tem como objetivo fazer uma revisão de alguns aspectos englobando: história, sintomatologia, tratamentos e modelos experimentais da esquizofrenia.
\end{abstract}

Descritores: Esquizofrenia. Neurotransmissores. Neuropsicologia.

\section{Histórico}

$\mathrm{O}$ histórico conceitual da esquizofrenia data do final do século XIX e da descrição da demência precoce por Emil Kraepelin. Outro cientista que teve importante influência sobre o conceito atual de esquizofrenia foi Eugen Bleuler.

Kraepelin (1856-1926) estabeleceu uma classificação de transtornos mentais que se baseava no modelo médico. Seu objetivo era delinear a existência de doenças com etiologia, sintomatologia, curso e resultados comuns. Ele chamou uma dessas entidades de demência precoce, porque começava no início da vida e quase invariavelmente levava a problemas psíquicos. Seus sintomas característicos incluíam alucinações, perturbações em atenção, compreensão e fluxo de pensamento, esvaziamento afetivo e sintomas catatônicos. A etiologia era endógena, ou seja, o transtorno surgia devido a causas internas.

1 Docente do Laboratório de Psicologia Experimental e Estudos da Cognição do Departamento de Ciências da Saúde da Escola Paulista de Medicina - UNIFESP. Endereço eletrônico: newstein@hotmail.com 
A demência precoce foi separada do transtorno maníaco-depressivo e da paranóia com base em critérios relacionados aos seus sintomas e curso. Kraepelin distinguiu três formas do transtorno: hebefrênica, catatônica e paranóide.

Bleuler (1857-1939) criou o termo "esquizofrenia" (esquizo = divisão, phrenia $=$ mente) que substituiu o termo demência precoce na literatura. Bleuler conceitualizou o termo para indicar a presença de um cisma entre pensamento, emoção e comportamento nos pacientes afetados. Para explicar melhor sua teoria relativa aos cismas mentais internos nesses pacientes, Bleuler descreveu sintomas fundamentais (ou primários) específicos da esquizofrenia que se tornaram conhecidos como os quatro "As": associação frouxa de idéias, ambivalência, autismo e alterações de afeto. Bleuler também descreveu os sintomas acessórios, (ou secundários), que incluíam alucinações e delírios (Ey, Bernard, \& Brisset, 1985).

\section{Subtipos}

Os três primeiros subtipos clássicos (demência paranóide, hebefrenia e catatonia) eram descritos como doenças separadas até que Kraepelin as reuniu sob o nome de demência precoce. Juntamente com a esquizofrenia simples, introduzida por Bleuler, os subtipos paranóide, hebefrênico e catatônico de Kraepelin formaram o grupo de esquizofrenias de Bleuler.

Diversos avanços, incluindo: a definição objetiva e uniformização dos sintomas e critérios diagnósticos para esquizofrenia; o advento das drogas antipsicóticas e a caracterização de seus mecanismos de ação e padrões de respostas; e, por fim, a identificação de anormalidades biológicas na doença, tem em conjunto, tornado possível o surgimento de classificações mais precisas de subtipos esquizofrênicos, que levam em conta, características de curso, resposta a tratamento, prognóstico e substrato patogênico. Dentre estas, a dicotomização da esquizofrenia em subtipos I e II ou positivo/negativo proposta por Crow (1980) é talvez a classificação mais conhecida. Segundo ele, os dois tipos podem refletir dois processos patológicos etiológica e prognosticamente distintos. Os principais sintomas da síndrome positiva (ou tipo I) são alucinações e delírios e da síndrome negativa (ou tipo II) são o embotamento afetivo e a pobreza do discurso. 


\section{Sintomas característicos}

Os primeiros sinais e sintomas da doença aparecem mais comumente durante a adolescência ou início da idade adulta. Apesar de poder surgir de forma abrupta, o quadro mais freqüente se inicia de maneira insidiosa. Sintomas prodrômicos pouco específicos, incluindo perda de energia, iniciativa e interesses, humor depressivo, isolamento, comportamento inadequado, negligência com a aparência pessoal e higiene, podem surgir e permanecer por algumas semanas ou até meses antes do aparecimento de sintomas mais característicos da doença. Familiares e amigos em geral percebem mudanças no comportamento do paciente, nas suas atividades pessoais, contato social e desempenho no trabalho e/ou escola (Vallada Filho \& Busatto Fillho, 1996).

Os aspectos mais característicos da esquizofrenia são alucinações e delírios, transtornos de pensamento e fala, perturbação das emoções e do afeto, déficits cognitivos e avolição.

Alucinações e delírios são freqüentemente observados em algum momento durante o curso da esquizofrenia. As alucinações visuais ocorrem em $15 \%$, as auditivas em $50 \%$ e as táteis em $5 \%$ de todos os sujeitos, e os delírios em mais de $90 \%$ deles (Pull, 2005).

O termo transtorno do pensamento refere-se a uma doença no conteúdo, assim como na forma dos pensamentos do indivíduo. Os transtornos do conteúdo do pensamento são os delírios. Os transtornos na forma de pensamento podem ser subdivididos em duas categorias: perturbação intrínseca do pensamento e, transtorno na forma em que os pensamentos são expressos na linguagem e na fala. A linguagem e o discurso desordenados descarrilamento, tangencialidade, neologismos, pobreza no conteúdo do discurso, incoerência, pressão da fala, fuga de idéias e fala retardada ou mutismo.

Os distúrbios do comportamento na esquizofrenia incluem comportamento grosseiramente desordenado e comportamento catatônico. Desde o começo, o comportamento catatônico foi descrito entre os aspectos característicos da esquizofrenia. A catatonia é definida como um conjunto de movimentos, posturas e ações complexas cujo denominador comum é a sua involuntariedade. Os fenômenos catatônicos incluem: estupor, catalepsia, automatismo, maneirismos, esteriotipias, fazer posturas e caretas, negativismo e ecopraxia. 
Foram encontrados sintomas catatônicos entre 5 e $10 \%$ dos pacientes com esquizofrenia. Entretanto, esses sintomas não são específicos da esquizofrenia, podendo ocorrer, sobretudo na mania (Pull, 2005).

A anedonia ou perda da capacidade de sentir prazer, foi proposta como a característica central ou cardinal da esquizofrenia. A anedonia física abrange a perda de prazeres como admirar a beleza do pôr-do-sol, comer, beber, cantar, ser massageado. A anedonia social abrange a perda de prazeres como estar com os amigos ou estar com outras pessoas. $\mathrm{O}$ embotamento afetivo foi considerado comum, mas não onipresente, em pacientes com esquizofrenia, sendo também comum em pacientes depressivos. Os déficits cognitivos foram relacionados como características inportantes da esquizofrenia desde as descrições originais de Kraepelin e Bleuler.

Pacientes com esquizofrenia demonstram um déficit cognitivo generalizado, ou seja, eles tendem a ter um desempenho em níveis mais baixos do que controles normais em uma variedade de testes cognitivos. Eles apresentam múltiplos déficits neuropsicológicos em testes de raciocínio conceitual complexo, velocidade psicomotora, memória de aprendizagem nova e incidental e habilidades motoras, sensoriais e perceptuais. As alterações cognitivas seletivas mais proeminentes na esquizofrenia incluem déficits em atenção, memória e resolução de problemas.

\section{Epidemiologia}

Há dezenas de estudos de prevalência em esquizofrenia realizados nos cinco continentes. Sua taxa é ao redor de 0,9-11 por 1.000 habitantes. Entretanto, os trabalhos de incidência em esquizofrenia são em menor número. Tais estudos são mais trabalhosos, requerem uma avaliação longitudinal, com duas avaliações em diferentes períodos sobre a mesma população e, a partir dessas avaliações, determinar quantos novos casos aparecem nesse mesmo intervalo. Sua incidência anual está entre 0,1-0,7 novos casos para cada 1.000 habitantes. 


\section{Teorias Etiológicas}

As causas da esquizofrenia são ainda desconhecidas. Porém, há consenso em atribuir a desorganização da personalidade, verificada na esquizofrenia, à interação de variáveis culturais, psicológicas e biológicas, entre as quais destacam-se as de natureza genética.

\section{Teoria Genética}

A esquizofrenia é uma desordem hereditária. Possuir um parente com esquizofrenia é o fator de risco mais consistente e significativo para o desenvolvimento da doença. Uma relação clara pode ser encontrada entre o risco de esquizofrenia e o grau de parentesco a uma pessoa com esquizofrenia. Muitos estudos epidemiológicos mostram que indivíduos que possuem parentes em primeiro grau com esquizofrenia possuem um risco aumentado em desenvolver a doença. Embora as estimativas variem, a taxa de concordância para esquizofrenia em gêmeos idênticos é ao redor de $50 \%$ e, para gêmeos dizigóticos, é da ordem de $12 \%$, sendo significativamente maior que o $1 \%$ de risco da população geral. Por um lado, estes estudos demonstram a existência do componente genético (quatro vezes maior a probabilidade em gêmeos monozigóticos do que em dizigóticos), mas, por outro lado, também ressalta a participação do componente ambiental na expressão da esquizofrenia, já que o esperado é teoricamente $100 \%$ de concordância para gêmeos monozigóticos, pois a carga genética é idêntica em ambos (Vallada Filho \& Busatto Fillho, 1996).

\section{Teorias Neuroquímicas}

Embora existam várias hipóteses bioquímicas desenvolvidas para explicar a gênese da esquizofrenia, nos deteremos naquela referente a hiperfunção dopaminérgica central, atualmente a mais bem investigada e mais aceita. No entanto, sabe-se que além do sistema dopaminérgico, outros sistemas de neurotransmissores centrais desempenham algum papel, sendo provável que vários sistemas estejam envolvidos simultaneamente (Lieberman, Mailman, \& Duncam, 1998). 


\section{A hipótese dopaminérgica}

A teoria dopaminérgica da esquizofrenia foi baseada na observação de que certas drogas tinham habilidade em estimular a neurotransmissão da dopamina (DA). Constatou-se que a droga psicoestimulante anfetamina, quando administrada em doses altas e repetidas, causa uma psicose tóxica com características muito semelhantes às da esquizofrenia paranóide em fase ativa. A semelhança é tão grande, que pode levar a erros diagnósticos, caso o psiquiatra ignore que o paciente tenha ingerido anfetamina. Sabe-se que essa droga atua nos terminais dopaminérgicos aumentando a liberação de DA, além de impedir sua inativação na fenda sináptica, por inibir o mecanismo neuronal de recaptação existente na membrana pré-sináptica. Assim, é possível que os sintomas esquizofreniformes - grande agitação psicomotora, alucinações auditivas, e idéias delirantes do tipo persecutório - sejam devidos ao excesso de atividade dopaminérgica determinado pela anfetamina. Com efeito, essas manifestações cedem rapidamente após a administração de neurolépticos bloqueadores dos receptores dopaminérgicos, sobretudo do tipo $\mathrm{D}_{2}$, ricamente distribuídos nos gânglios da base e áreas mesolímbicas. Além disso, alguns pacientes parkinsonianos tratados com L-DOPA, que aumenta a formação de DA, desenvolvem sintomas psicóticos semelhantes. Sabe-se ainda que o efeito antipsicótico de drogas como a clopromazina e o haloperidol deve-se a ação dessas drogas em bloquear a atividade dopaminérgica (Graeff, 1989, 2004).

\section{Esquizofrenia como Distúrbio do Neurodesenvolvimento}

Durante as últimas duas décadas, novas evidências científicas levaram a uma hipótese reformulada da patogênese da esquizofrenia baseada em processos relacionados ao desenvolvimento cerebral. Existem vários estudos sugerindo que eventos de ocorrência precoce, durante a vida intra-uterina ou logo após o nascimento, podem ser de importância fundamental na etiologia de uma parcela dos casos de esquizofrenia, interferindo no desenvolvimento normal de determinadas estruturas cerebrais e tornando o indivíduo vulnerável ao surgimento mais tardio dos sintomas da doença. A má nutrição do feto, envolvendo especialmente redução no suprimento de oxigênio, iodo, glicose, e ferro podem levar a prejuízos no desenvolvimento do sistema nervoso central (SNC). As condições maternas que causam a má nutrição do feto e parecem 
aumentar o risco de desenvolvimento da esquizofrenia incluem diabete, doença pulmonar crônica, anemia, inanição maternal durante o primeiro trimestre de gestação (Akil \& Weinberger, 2000).

O nascimento prematuro extremo (ex. antes de 33 semanas gestacionais) parece aumentar o risco para a esquizofrenia.

Eventos perinatais e complicações durante o parto podem causar danos ao hipocampo e córtex cerebral por causar hipóxia ou isquemia. Assim, eventos que afetam o desenvolvimento cerebral durante a gestação e o período perinatal estão claramente relacionados à esquizofrenia. Entretanto, inúmeras restrições devem ser feitas. Embora significativos esses efeitos são de uma pequena magnitude e podem explicar apenas uma pequena porcentagem dos casos. Além disso, a perturbação do meio intra-uterino que tem sido ligado à esquizofrenia também pode aumentar os riscos para ocorrência de outros transtornos psiquiátricos (Akil \& Weinberger, 2000).

\section{Alterações Estruturais}

Por volta da metade da década de 1970, diversos estudos utilizando a recém-desenvolvida técnica de tomografia computadorizada (TC) de crânio passaram a detectar sinais consistentes de atrofia cerebral, incluindo alargamento dos ventrículos cerebrais (mais pronunciado em cornos posteriores) e aumento de sulcos corticais numa proporção considerável de pacientes esquizofrênicos crônicos e agudos, independentemente de fatores não-específicos como idade, uso de neurolépticos e eletroconvulsoterapia (ECT). Os achados pioneiros de TC de crânio renovaram também o interesse em estudos patológicos post-mortem em cérebros de esquizofrênicos, e, com os avanços metodológicos nesta área, novas evidências começaram a surgir. Estudos recentes sugerem que os cérebros de alguns pacientes esquizofrênicos são mais leves e menores em comparação a indivíduos normais. Além disso, alterações cerebrais mais localizadas têm sido identificadas. As áreas mais consistentemente implicadas têm sido as porções mediais dos lobos temporais, sobretudo hipocampo e giro para-hipocampal. Diminuições de volume em áreas frontais, tálamo, gânglios da base e corpo caloso têm sido também sugeridas. A descoberta de alterações estruturais nos lobos temporais mediais tem despertado particular interesse. A idéia de que alterações nestas áreas estão relacionadas 
com a gênese de sintomas esquizofrênicos já vinha sendo considerada uma vez que psicoses esquizofreniformes são freqüientes em pacientes com epilepsia do lobo temporal (Hopkins \& Lewis, 2000).

A partir da década de 1980, estudos usando ressonância magnética nuclear (RMN), técnica de imageamento mais sofisticada que a TC, têm permitido a avaliação de pacientes esquizofrênicos vivos e não medicados com excelente resolução anatômica. Os achados de RMN confirmam a presença de reduções volumétricas especificamente em lobos temporais mediais, mais pronunciadas no hemisfério esquerdo. Outras áreas temporais têm sido também implicadas, principalmente aquelas envolvidas em produção de linguagem, como o giro temporal superior. A consistência dos achados de RMN e neuropatalógicos tem levado diversos pesquisadores a sugerir que alterações estruturais nos lobos temporais estão invariavelmente presentes na doença, variando em intensidade de paciente para paciente.

\section{Teorias Psicológicas}

A partir da década de 40, teorias psicológicas tentando explicar a esquizofrenia a partir de relacionamentos familiares patológicos e padrões de comunicação interpessoal aberrantes ganharam força, influenciando certas escolas do pensamento psiquiátrico. Em 1948, Fromm-Reichmann introduziu a idéia da "mãe esquizofrenogênica", observando que determinados padrões de comportamento materno, incluindo hostilidade e rejeição, pareciam ser freqüentes em mães de pacientes que desenvolviam esquizofrenia, entretanto a falta de comprovação empírica fez com que caísse em desuso.

Mais recentemente, os avanços metodológicos que tanto têm contribuído para o apuro dos critérios diagnósticos de esquizofrenia e para o esclarecimento das bases biológicas da doença, passaram também a ser aplicados na investigação dos aspectos psicossociais associados à mesma. Duas frentes em particular têm demonstrado, de forma convincente, que, se fatores psicossociais não estão primariamente relacionados com a etiologia da doença, certamente influenciam a forma de aparecimento e o curso dos sintomas esquizofrênicos.

Uma destas correntes diz respeito ao ambiente familiar de pacientes esquizofrênicos. O conceito de emoção expressa (EE) tornou-se particular- 
mente importante nesta área, sendo usado para definir determinados tipos de atitudes hostis ou do excesso de envolvimento emocional por parte de familiares de esquizofrênicos. Estudos utilizando metodologia adequada, (entrevistas bem estruturadas) para avaliar a presença destas atitudes, têm demonstrado que altos índices de EE na família aumentam significativamente a taxa de recaída e reinternação hospitalar de esquizofrênicos. Apesar do mecanismo pelo qual a EE leva a recaída ser ainda desconhecido, os achados anteriores têm implicações fundamentais não só para o esclarecimento da relação fatores psicossociais versus sintomas esquizofrênicos, mas também para a formulação de estratégias terapêuticas e psicoeducativas visando a prevenção de recaídas (Brown, Birley, \& Wing, 1972).

A Segunda corrente atual de pesquisa na área relaciona-se com o estudo da influência de "eventos estressores psicossociais" no curso da doença. Sabe-se que o curso de diversas doenças de clara etiologia biológica pode ser influenciado por "eventos estressores psicossociais" como perda de familiar próximo, mudança de moradia, exames escolares etc. Estudos recentes investigando a influência destes life-events no curso da esquizofrenia sugerem que pacientes esquizofrênicos podem apresentar pioras sintomatológicas diante deste tipo de estresse (Zubin \& Spring, 1977).

Diversos modelos têm procurado integrar os aspectos psicossociais descritos anteriormente aos aspectos biológicos da etiologia da esquizofrenia. Dentre estes, o modelo da "vulnerabilidade versus estresse" parte do princípio que pacientes esquizofrênicos apresentam uma vulnerabilidade para a doença de caráter biológico (genética e/ou decorrentes de insultos ambientais precoces), mas reconhecem também que o deflagrar dos sintomas pode ser diretamente influenciado pelo grau de estresse psicossocial ao qual o indivíduo é submetido. Dentro deste prisma, forma, intensidade e curso dos sintomas esquizofrênicos são vistos como um balanço entre o grau de vulnerabilidade biológica do paciente e a intensidade de estresse ambiental. O modelo "vulnerabilidade - estresse psicossocial" integra aspectos biológicos e psicossociais da doença, e justifica a necessidade de uma abordagem mais global ao tratamento do paciente esquizofrênico, levando em conta não só a eliminação dos sintomas, mas também o controle e prevenção de fatores ambientais estressores. 


\section{Tratamento}

\section{Tratamento Farmacológico com Antipsicóticos}

\section{Antipsicóticos Típicos}

Foi em meados do século XX que se iniciou uma profunda transformação no setor da Medicina que trata das chamadas doenças mentais. Ela ficou conhecida como a Revolução Farmacológica da Psiquiatria, pois se deveu basicamente à introdução dos medicamentos psicoterapêuticos, capazes de melhorar consideravelmente o estado de muitos pacientes portadores de alterações acentuadas de diversas funções psicológicas e perda do juízo da realidade. Em Psiquiatria, tais desordens são denominadas psicoses. $\mathrm{O}$ termo psicose descreve transtornos psiquiátricos graves, geralmente de origem desconhecida ou idiopática (funcionais). Nestes quadros são encontrados, além de alterações do comportamento, incapacidade de pensar coerentemente e de compreender a realidade. A orientação e a memória estão conservadas, apesar do comprometimento do pensamento e das emoções (Wyatt, 2001).

Até o início dos anos cinqüenta, os recursos de que se dispunha para tratar os doentes psicóticos eram muito limitados e a única solução era confiná-los em grandes hospitais ou asilos, de onde muitos não podiam mais sair. $\mathrm{O}$ fator fundamental dessa mudança foi a introdução dos medicamentos antipsicóticos, também conhecidos como neurolépticos, ou tranquilizantes maiores.

A descoberta dos neurolépticos, no ano de 1952, marca o início da Psicofarmacologia contemporânea. Nessa época, o cirurgião francês Henri Laborit utilizava uma mistura de drogas, que denominava "coquetel lítico", para abrandar reações neurovegetativas de pacientes submetidos a cirurgias prolongadas realizadas a baixas temperaturas - a assim chamada "hibernação artificial". Desta mistura participava o composto anti-histamínio prometazina. Ao observar os efeitos de seu análogo químico, clorpromazina, Laborit notou que os pacientes ficavam em estado peculiar, que passou a ser conhecido como síndrome neuroléptica, caracterizada por indiferença emocional, sem diminuição importante da vigilância. Esta observação casual despertou a curiosidade de Laborit, que sugeriu a dois colegas psiquiatras, Jean Delay e Pierre Deniker, o uso experimental da droga em pacientes mentais. Como conseqüência, 
a clorpromazina foi administrada a pacientes internados por longo tempo em hospitais psiquiátricos. Surpreendentemente, muitos desses pacientes apresentaram melhora considerável, e puderam mesmo retornar ao convívio social. Em especial, os sintomas psicóticos característicos da esquizofrenia eram aliviados após algum tempo de uso da droga. A notícia logo se propagou, e o uso da clorpromazina e de seus análogos difundiu-se pelo mundo. Como resultado, a prática psiquiátrica mudou muito, passando pela chamada revolução farmacológica. Sobretudo, o número de leitos dedicados a pacientes crônicos passou a diminuir a partir de meados da década de 1950, nas nações mais desenvolvidas. Nelas, o uso do tratamento farmacológico abriu portas para intervenções sociais e psicológicas, que trazem benefício adicional ao paciente (Graeff \& Guimarães, 1999).

A utilização da clorpromazina revolucionou o tratamento dos pacientes esquizofrênicos, já que resultou em melhora significativa de $50-75 \%$ e quase $90 \%$ destes indivíduos apresentaram algum beneficio clínico decorrente do uso deste fármaco. Seguindo-se a clorpromazina, foram descobertos diversos outros compostos com propriedades farmacológicas semelhantes, embora com estrutura química diferente.

Os antipsicóticos levam dias a semanas para produzirem seus efeitos terapêuticos máximos. Como vimos, sua principal ação consiste no bloqueio de receptores dopaminérgicos $\mathrm{D}_{2}$ no sistema nervoso central. Diversos estudos demonstram uma relação direta entre a potência terapêutica dessas drogas e sua capacidade em bloquear receptores $\mathrm{D}_{2}$ (Snyder, 1986).

Os neurolépticos podem ser divididos em dois grupos de acordo com sua potência. Assim, temos os antipsicóticos de "alta potência" (como o haloperidol, flufenazina, trifluoperazina, tiotixene) e os de "baixa potência" ou sedativos (como clorpromazina, tioridazina etc).

Entre os sistemas dopaminérgicos cerebrais nos quais os antipsicóticos atuam, o mesocortical e o mesolímbico são os que provavelmente estão mais relacionados com a fisiopatologia da esquizofrenia. Já o bloqueio de receptores dopaminérgicos do sistema nigroestriatal é considerado responsável pelos efeitos extrapiramidais dos neurolépticos, enquanto a ação no sistema túberoinfundibular parece ser responsável pelos efeitos endocrinológicos causados por estas dogras (Graeff \& Guimarães, 1999), (Tabela 1). 
Tabela 1: Vias dopaminérgicas centrais e suas ações.

\begin{tabular}{ll}
\hline VIAS & FUNÇÕES \\
\hline Mesolímbica & $\begin{array}{l}\text { Relacionada a comportamentos e emoções. Sua } \\
\text { hiperatividade relaciona-se aos sintomas } \\
\text { psicóticos positivos. }\end{array}$ \\
Mesocortical & $\begin{array}{l}\text { O papel na mediação de sintomas psicóticos } \\
\text { ainda é controverso, mas parece que seu bloqueio } \\
\text { associa-se a piora de sintomas negativos. }\end{array}$ \\
Nigroestriada & $\begin{array}{l}\text { Coordenação de movimentos voluntários. Seu } \\
\text { bloqueio está associado a distúrbios motores com } \\
\text { acatisia, distonia, tremores, rigidez e } \\
\text { acinesia/bradicinesia e em longo prazo pode levar } \\
\text { a discinesia tardia. }\end{array}$ \\
Tuberoinfundibular & $\begin{array}{l}\text { Relacionada a inibição da secreção de prolactina. } \\
\text { Seu bloqueio associa-se a aumento dos níveis } \\
\text { desse hormônio. }\end{array}$ \\
\hline Meduloperiventricular & \begin{tabular}{l} 
Relacionado com o comportamento alimentar \\
\hline
\end{tabular}
\end{tabular}

Infelizmente, os antipsicóticos não fazem mais que atenuar a intensidade das manifestações psicóticas agudas, sendo incapazes de curar o paciente. São fundamentalmente efetivos no tratamento dos sintomas positivos da doença, sendo questionável sua ação sobre sintomas negativos. Entretanto, centenas de ensaios clínicos controlados comprovam que o tratamento de manutenção, a longo prazo, com neurolépticos reduz drasticamente a freqüência de recidivas e reospitalizações.

\section{Efeitos colaterais}

Além do efeito terapêutico, os neurolépticos causam efeitos colaterais típicos. Como vimos, a via dopaminérgica nigroestriatal participa da regulação da atividade motora. Por bolquearem receptores dopaminérgicos estriatais, os antipsicóticos típicos podem produzir o aparecimento de efeitos adversos 
extrapiramidais. Estes incluem a síndrome de Parkinson, reações distônicas agudas, acatisia, acinesia e síndrome neuroléptica maligna. A incidência desses efeitos é bastante elevada, chegando até a $90 \%$ em alguns estudos, e costuma ocorrer nas primeiras semanas de tratamento. A síndrome de Parkinson caracteriza-se por lentidão dos movimentos (bradicinesia), tremor variável das extremidades (aumenta com a movimentação e está abolido no sono), imobilidade da expressão facial, alteração da marcha e postura rígida. Reações distônicas agudas também podem ocorrer, como espasmos dos músculos da face, pescoço e língua. A acatisia refere-se ao estado de desconforto intenso nos membros inferiores, acompanhado de incapacidade de ficar com as pernas paradas. No entanto, o efeito adverso motor mais temido dos neurolépticos é a discinesia tardia. Ela é caracterizada por movimentos esteriotipados involuntários, principalmente da face, como sucção com os lábios, movimentos laterais da mandíbula e movimentos anormais da língua, descritos como flycatching. Pode também envolver movimentos coreiformes de braços, troncos ou pernas. Esses movimentos aumentam em estados de alerta e diminuem com o repouso. A discinesia tardia pode aparecer após anos de tratamento com neurolépticos, dependendo da dose e da duração do tratamento. Sua incidência geral é estimada em 10\%-20\% dos pacientes crônicos, mas pode ser maior que $50 \%$ em pacientes com mais de 60 anos (Graeff \& Guimarães, 1999).

Outros efeitos adversos também de origem central são os que afetam o sistema neuroendócrino causando ginecomastia, galactorréia e amenorréia. Os neurolépticos atuam também na periferia do organismo, afetando o sistema nervoso autônomo. Assim, bloqueiam os receptores da acetilcolina, do tipo muscarínico, levando a sintomas como secura da boca e da pele, midríase e dificuldade de acomodação visual, taquicardia, constipação intestinal e retenção urinária.

\section{Antipsicóticos Atípicos}

Atualmente, os esforços concentram-se na busca de antipsicóticos com menos efeitos extrapiramidais e que sejam eficazes no tratamento dos sintomas negativos da esquizofrenia, denominados antipsicóticos atípicos, a exemplo da clozapina, risperidona, olanzapina, quetiapina, ziprasidona e mais recentemente o aripiprazol. 
O desenvolvimento da clozapina foi um claro avanço nesses esforços. Essa substância, disponível em alguns países da Europa desde o começo da década de 1970, e introduzida nos EUA na década de 1990, foi o primeiro antipsicótico que tratou os sintomas da esquizofrenia de forma efetiva com apenas um risco mínimo de induzir efeitos colaterais motores extrapiramidais. Além de mudar as opiniões em relação à eficácia clínica e aos efeitos adversos dos fármacos, o sucesso da clozapina também influenciou de forma notável as estratégias pré-clínicas para o desenvolvimento de novos antipsicóticos. De modo geral, isso foi alimentado pelo reconhecimento de que a clozapina tem eficácia antipsicótica excelente, em um grau semelhante aos neurolépticos clássicos, sem bloquear os receptores de dopamina $\mathrm{D}_{2}$ nigrostriatais. Até esse ponto, considerava-se que um forte bloqueio dos receptores $\mathrm{D}_{2}$ era pré-requisito para o efeito antipsicótico (Fleischhacker, 2005).

Na clínica, a clozapina é igual ou superior aos antipsicóticos clássicos na melhoria dos sintomas positivos da esquizofrenia, mas também mostra efeitos em sintomas negativos. Além disso, $60 \%$ dos pacientes que não respondem a neurolépticos típicos podem apresentar melhora com o seu uso. O maior problema desta droga, no entanto, é o aparecimento eventual de agranulocitose. Esse quadro, de origem controversa, talvez com componentes alérgicos, caracteriza-se pela redução acentuada do número de neutrófilos, tornando o paciente susceptível a infecções graves. A monitorização do uso da droga por intermédio de hemogramas regulares faz-se necessária (Graeff \& Guimarães, 1999).

\section{Tratamento Psicoterápico}

Com a introdução dos neurolépticos na década de 1950, a quantidade de pacientes internados diminuiu rapidamente. Infelizmente, pacientes bastante incapacitados muitas vezes eram devolvidos à sociedade despreparados para enfrentar as demandas das integrações social e profissional. Dentro desta perspectiva, a farmacoterapia antipsicótica deve fazer parte de uma abordagem terapêutica ampla e abrangente visando à reabilitação psicossocial do paciente. Enquanto a medicação neuroléptica pode reduzir os sintomas positivos e prevenir recaídas psicóticas, o apoio psicoterapêutico e o treinamento de estratégias de enfrentamento e manejo de situações de vida ajudam o paciente 
a adaptar-se ao ambiente e a enfrentar o estresse, sendo que as intervenções familiares e sócio-profissionais modificam fatores ambientais de acordo com a capacidade do paciente.

Essa abordagem multidimensional é oferecida, avaliada e coordenada continuamente ao longo do tempo em contextos diferentes adaptados para cada paciente e suas necessidades específicas. Dessa forma, pode-se evitar o risco de provocar a exacerbação de sintomas psicóticos por estimulação exagerada ou induzir a regressão e o déficit por baixa estimulação.

Antes da década de 1970, as psicoterapias individuais e de grupo para a esquizofrenia baseavam-se geralmente em teorias psicodinâmicas ou, ainda, em teorias que consideravam que a esquizofrenia seria causada por padrões patogênicos de comportamento ou de comunicação da família do indivíduo. Além de não serem efetivos em termos dos sintomas psicóticos fundamentais, esses métodos de tratamento estigmatizavam as famílias dos pacientes, que muitas vezes eram o seu principal sistema de apoio. Assim, após a introdução de antipsicóticos efetivos na década de 1960, houve afastamento do foco nas intervenções psicológicas para a esquizofrenia (McGlashan, 1994).

Gradualmente houve insatisfação com a confiança exagerada nos tratamentos farmacológicos para a esquizofrenia, e uma proporção elevada de indivíduos com esquizofrenia continuava a experimentar sintomas psicóticos positivos apesar de tomar antipsicóticos. De maneira semelhante, verificou-se que a utilidade dessa medicação para remediar os prejuízos cognitivos e sociais associados à doença era limitada. Além disso, em uma série de estudos que atualmente são considerados clássicos, Brown et al. (1972) demonstraram que a natureza do ambiente familiar para o qual o paciente esquizofrênico retorna após a alta hospitalar é fortemente preditiva de recaídas psicóticas no decorrer dos nove meses seguintes. Assim, na década de 1980, surgiram ensaios controlados de intervenções psicológicas projetadas para promover a aquisição de habilidades sociais e reduzir recaídas, melhorando a atmosfera familiar. Seus resultados positivos foram bem-recebidos e promoveram um interesse maior nas terapias psicológicas para essa população (Birchwood \& Spencer, 2005).

Concomitante a esses acontecimentos, a utilização da terapia cognitiva para os sintomas da depressão encorajou os médicos e pesquisadores a estenderem suas técnicas ao tratamento de sintomas psicóticos positivos resistentes 
à medicação. $\mathrm{O}$ uso de uma intervenção cognitiva para sintomas psicóticos da esquizofrenia foi relatado por Beck já em 1952. Ele utilizou uma variedade de técnicas cognitivas (como o exame cuidadoso dos antecedentes dos sintomas, teste da realidade e exercícios de tarefa de casa) no tratamento de um paciente com esquizofrenia paranóide. Essas técnicas formam a base da abordagem cognitivo-comportamental atual para o tratamento individual de sintomas psicóticos. As intervenções psicológicas afastaram-se do foco em classes tradicionais de diagnóstico psiquiátrico, voltando-se para um foco detalhado nos fenômenos da experiência e do comportamento associados à esquizofrenia. Intervenções sobre sintoma único foram combinadas com estratégias globais de tratamento como parte do desenvolvimento da terapia cognitivo-comportamental, que veio acompanhada por uma necessidade de maior precisão na medida de sintomas individuais para planejar tratamentos. Inicialmente surgiram resultados promissores em relatos de casos e estudos não-controlados, e, nos últimos anos, diversos ensaios clínicos controlados randomizados foram publicados. Também recentemente, a atenção concentrou-se no tratamento da depressão causada pela esquizofrenia.

\section{Modelos experimentais de esquizofrenia}

Modelo de doença é qualquer preparação experimental desenvolvida para estudar quaisquer aspectos de determinada doença, inclusive métodos terapêuticos. O modelo pode ser desenvolvido em indivíduos humanos, em animais de laboratório ou mesmo em culturas de células e até em simulações matemáticas no computador.

Os modelos experimentais de doenças mentais foram desenvolvidos para tentar prever a ação terapêutica de um determinado fármaco, mesmo que os mecanismos fisiopatológicos fossem quase nada compreendidos. Nesse contexto, Paul Willner propôs, em 1984, critérios crescentes e hierárquicos, conforme o tipo de validade que o modelo pode atingir. São eles: a validade preditiva (predictive validity), a validade fenomenológica (face validity) e a validade por constructo (construct validity). A validade preditiva é considerada o nível mais baixo de validação e corresponde ao isomorfismo farmacológico, ou seja, todas as manipulações farmacológicas - especialmente as terapêuti- 
cas, que sabidamente influem na doença - devem ter efeito similar no modelo. A validade fenomenológica requer isomorfismo sintomático entre a doença e o modelo. Por último, a validade por constructo, o nível mais alto de validação, requer a existência de constructos teóricos que relacionem o modelo e a fisiopatologia da doença. A validação resultaria da definição da variável a ser estudada e da integração dela aos dados comportamentais, evolutivos, neuropatológicos e farmacológicos.

As dificuldades próprias de um modelo de esquizofrenia que abranja toda a complexidade da doença parece irrealizável. A esquizofrenia provavelmente tem múltipla etiologia, envolvendo interações entre fatores genéticos e ambientais e produzindo complexa disfunção nervosa. Além disso, envolve funções superiores, como abstração e linguagem, impossíveis de serem acessadas em animais. Talvez, por isso as tentativas de modelos experimentais causaram pouco impacto e algum ceticismo.

A impossibilidade de reproduzir experimentalmente todo o fenótipo da esquizofrenia, entretanto, está longe de indicar que se deva abandonar esse campo de pesquisa. Os pesquisadores estão mudando do foco holístico para modelos de fenótipos quantitativos, chamados também de endofenótipos ou traços intermediários e pela respectiva manipulação de seus correlatos experimentais, feita por intervenções específicas (ex. farmacológicas, cirúrgicas, genéticas) na busca de um mecanismo comum para estes endofenótipos (Salgado, Hetem, \& Sandner, 2006).

Endofenótipos estudados nos modelos de esquizofrenia

Inibição por pré-pulso do reflexo de sobressalto

O modelo de inibição por pré-pulso (IPP) do reflexo de sobressalto ocorre quando um estímulo fraco (pré-pulso), geralmente acústico, é apresentado em um intervalo de 10 até $200 \mathrm{~ms}$ antes de um estimulo acústico forte (pulso), reduzindo, dessa forma, a magnitude da resposta de sobressalto. A função da IPP é a de filtrar informações irrelevantes, possibilitando ao indivíduo direcionar sua atenção a aspectos mais importantes do ambiente refletindo dessa forma um processo pré-atentivo. Em todas as espécies de mamíferos testadas, incluindo a humana, o pré-pulso reduz ou impede a resposta de so- 
bressalto causada pelo pulso. A abolição da IPP poderia ser explicada, portanto, pela incapacidade para prestar atenção ao pré-pulso, de tal modo que poderia ocorrer em todas as condições que incluem prejuízo atencional. Mesmo não sendo específico da esquizofrenia, a IPP se relaciona a uma propriedade cognitiva que está na origem da doença ou que a compõe. Pacientes com esquizofrenia exibem déficits nestas respostas correlacionados entre indivíduos com sintomas cognitivos centrais incluindo desordem do pensamento e déficit atencional e com sintomas comportamentais incluindo sintomas positivos e negativos (Rosa et al., 2004).

O modelo da IPP tem sido sistematicamente utilizado para investigar os substratos neurais e farmacológicos relacionados à esquizofrenia (Silva, Sandner, \& Brandão, 2005).

Déficits similares na IPP podem ser reproduzidos em ratos por manipulações farmacológicas, através da estimulação de receptores dopaminérgicos $\mathrm{D}_{2}$, por anfetamina ou por apomorfina ou pelo bloqueio de receptores N-metil-D-aspartato (NMDA) (Geyer, Thomson, Braff, \& Swerdlow, 2001).

\section{Inibição latente}

A inibição latente (IL) é tradicionalmente definida como o "retardo" na aprendizagem associativa normalmente manifesta por um organismo quando um estímulo neutro é inicialmente apresentado desacompanhado de qualquer consequiência e em seguida utilizado como estímulo condicionado. Em outras palavras, a IL consiste em um decréscimo na capacidade de adquirir uma nova associação quando o organismo foi previamente exposto ao "estímulo a ser condicionado". Esse decréscimo tem sido atribuído a uma variedade de mecanismos que reduzem a atenção ao estímulo pré-exposto (Lubow, Weiner, \& Feldon, 1982).

A IL ocorre em várias espécies, inclusive no homem, e parece ter a função de proteger o organismo de estímulos sem significado para a sua sobrevivência. Como o organismo está recebendo, a todo momento, uma enorme quantidade de estímulos provenientes do meio, a IL funciona como um mecanismo de seleção de estímulos. Dessa forma, os estímulos irrelevantes não interferem no comportamento do organismo, sendo rapidamente desconsiderados e deixando o sistema livre para processar informações que possam ser relevantes e significativas para o organismo (Brandão, Troncoso, Melo, \& Sandner, 1997). 
A alteração da IL pode estar relacionada a problemas na codificação de informações complexas pela memória ou na acessibilidade de sua lembrança. Note-se que os pacientes com esquizofrenia sofrem de problemas mnésicos importantes, como a incapacidade de estabelecer ligações complexas entre suas lembranças, com importante prejuízo da memória episódica ou contextual.

\section{Habituação}

Habituação é a redução de respostas a um estímulo, quando este ocorre sem conseqüências, repetidas vezes. É facilmente testada em seres humanos e animais de laboratório. Trata-se de uma das formas mais simples de aprendizagem implícita e parece alterada em pacientes esquizofrênicos.

\section{Hiperlocomoção e esteriotipia}

Animais e indivíduos humanos sob efeito de anfetamina, fármaco que pode produzir sintomas psicóticos, exibem hiperatividade motora e esteriotipia. Tais comportamentos podem ocorrer na esquizofrenia, sobretudo o comportamento esteriotipado e as perseverações, embora possa ocorrer também a redução da atividade motora.

\section{Movimentos oculares anti-sacádicos}

Neste teste, o sujeito deve inicialmente olhar para um ponto fixo central na tela de um computador. Um estímulo é então apresentado à direita ou à esquerda do ponto fixo e o sujeito é instruído a olhar para o lado oposto ao estímulo (movimento anti-sacádico). Pacientes com esquizofrenia mostram maior tendência em olhar de início diretamente para este estímulo, o que provavelmente indica comprometimento do controle inibitório.

\section{Interação social}

O isolamento social é um dos sintomas negativos que aparece com muita freqüência e cedo no curso da esquizofrenia. Modelos para estudar a interação social em animais de laboratório, sob o efeito de fármacos psicotomiméticos, têm sido tentados, mas são limitados pela forte diferença com a interação humana, imposta pelo papel da linguagem nesta última. Os testes consistem, em geral, na medida da distância média entre os indivíduos ao longo do dia. 
Entre os endofenótipos avaliados nas diferentes abordagens, a diminuição da interação social é o único ligado à sintomatologia negativa da esquizofrenia.

\section{Desempenho em teste neuropsicológicos}

$\mathrm{Na}$ avaliação neuropsicológica dos pacientes com esquizofrenia, o comprometimento aparece, sobretudo, em tarefas de funções executivas, como as que requerem memória operativa, flexibilidade mental ou atenção sustentada, bem como em testes de memória explícita episódica ou semântica.

A avaliação dessas funções em animais de laboratório é desejável para a compreensão de seus mecanismos. Embora sua avaliação em ratos seja dificultada por sua limitada capacidade cognitiva, existem paradigmas que se propõem a avaliar constructos cognitivos comparáveis aos humanos, como modelos de memória operativa, utilizáveis nestes animais. $\mathrm{O}$ uso de primatas não-humanos, por outro lado, proporciona excelente abordagem para tentar elucidar as bases neurobiológicas das funções cognitivas.

O conjunto de dados provenientes dos modelos experimentais de esquizofrenia tem permitido a formulação de interessantes hipóteses sobre a fisiopatologia da doença e de transtornos correlatos, como a esquizotipia.

\section{Perspectivas}

A esquizofrenia é a patologia que mais desperta atenção e interesse em psiquiatria, e também a que mais exaustivamente tem sido estudada, em inúmeros de seus aspectos e sob diferentes pontos de vista pelo fato de ser um transtorno cerebral grave, duradouro e debilitante.

No estudo etiológico da esquizofrenia, é desejável o esclarecimento de dois aspectos. De um lado é necessário determinar como uma alteração discreta e de causas variadas no desenvolvimento do sistema nervoso pode converte-se em um conjunto característico de endofenótipos. Por outro lado, além das alterações que predispõem a doença, é necessário apontar os fatores do ambiente do qual o sistema nervoso sofrerá o efeito patogênico, seja o ambiente interno ao corpo, seja o ambiente externo. 
De forma clara, os conceitos modernos do manejo da esquizofrenia incluem medidas psicossociais e reabilitativas. Embora a farmacoterapia ainda seja a espinha dorsal de tratamento, ela deve sempre vir embutida em procedimentos de tratamento integrados, que incluem todos os níveis de intervenção.

Silva, R. C. B. (2006). Schizophrenia: a review. Psicologia USP, 17(4), 263-285.

\begin{abstract}
The current definition of schizophrenia indicates that it is a term reserved for idiopathic cases of chronic psychosis. It may prove to be a cluster of different illnesses, with similar and overlapping symptoms. Schizophrenia is of multifactorial origin. Both genetic and environmental factors have been associated with an increased risk for schizophrenia. This paper is a review of some aspects of the history, symptoms, treatments and experimental models of schizophrenia.
\end{abstract}

Index terms: Schizophrenia. Neurotransmitters. Neuropsychology.

Silva, R. C. B. (2006). Schizophrénie: une revision. Psicologia USP, 17(4), 263-285.

Résumé: La définition actuelle de la schizophrénie reste celle d'une psychose chronique idiopathique. Ce status quo pourrait être dû à la multiplicité des affections rassemblées sous un même diagnostic. Il pourrait s'agir d'une maladie multifactorielle dont la survenue dépendrait d'une prédisposition génétique et de facteurs environnementaux. Cet article se propose de montrer une revisión sur les aspects de la histoire, symptômes, traitements et modèles expérimentaux de la chizophrénie . Mots-clés: Schizophrénie. Neuro-transmetteurs. Neuropsychologie. 


\section{Referências}

Akil, M., \& Weinberger, D. (2000). Neuropathology and the neurodevelopmental model. In P. J Harrison \& G. W. Roberts (Eds.), The neuropathology of schizophrenia. Progress and interpretation (pp. 189-212). New York: Oxford University Press.

Beck, A. T. (1952). Successful out-patient psychoterapy of a chronic schizophrenic with a delusion based on borrowed guilt. Psychiatry, 25, 305-312.

Birchwood, M., \& Spencer, E. (2005). Psicoterapias para a esquizofrenia: uma revisão. In M. Maj \& N. Sartorius (Org.), Esquizofrenia (pp. 133-203). Porto Alegre: Artmed.

Brandão, M. L., Troncoso, A. C., Melo, L. L., \& Sandner, G. (1997). Active avoidance learning using brain stimulation applied to the inferior colliculus as negative reinforcementinrats: Evidence for latent inhibition. Neuropsychobiology, 35(1), 30-35.

Brown, G. W., Birley, J., \& Wing, J. (1972). Influence of family life on the course of schizophrenia: A replication. British Journal of Psychiatry, 21, 241-258.

Crow, T. J. (1980). Molecular pathology of schizophrenia. More than one disease process? British Medical Journal, 280, 66-69.

Ey, H., Bernard, P., \& Brisset, C. (1985). As psicoses esquizofrênicas. In Manual de psiquiatria (pp. 535-615). Rio de Janeiro: Masson.

Fleischhacker, W. W. (2005). Tratamento farmacológico da esquizofrenia: uma revisão. In M. Maj, \& N. Sartorius (Org.), Esquizofrenia (pp. 71-132). Porto Alegre: Artmed.

Fromm-Reichmann, F. (1948). Notes of the development of treatment of schizophrenics by psychoanalytic psychotherapy. Psychiatry, 3, 263-273.

Geyer, M. A., Thomson, K. K., Braff, D., \& Swerdlow, N. (2001). Pharmacological studies of prepulse inhibition models of sensoriomotor gating deficits in schizophrenia: A decade in review. Psychopharmacology, 156, 117-154.

Graeff, F. G. (1989). Antipsicóticos. In Drogas psicotrópicas e seu modo de ação (pp. 2139). São Paulo: E.P.U.

Graeff, F. G. (2004). Serotonin, the periaqueductal gray and panic. Neuroscience and Biobehavioural Reviews, 28(3), 239-259.

Graeff, F. G., \& Guimarães, F. S. (1999). Medicamentos antipsicóticos. In F. G. Graeff, F. S. Guimarães \& A. V. Zuardi (Org.), Fundamentos de psicofarmacologia (pp. 69-91). São Paulo: Atheneu.

Hopkins, R., \& Lewis, S. (2000). Structural imaging findings and macroscopic pathology. In P. J. Harrison \& G. W. Roberts (Eds.), The neuropathology of schizophrenia. Progress and interpretation (pp. 5-56). New York: Oxford University Press. 
Lieberman, J.A., Mailman, R. B., \& Duncam, G. (1998). Serotoninergic basis of antipsychotic drug effects in schizophrenia. Biological Psychiatry, 44, 1099-1117.

Lubow, R. E., Weiner, I., \& Feldon, J. (1982). An animal model of attention. In M. Y. Spiegelstein \& A. Levy (Eds.), Behavioral models and the analysis of drugs action (pp. 89-107). New York: Elsevier.

McGlashan, T. H. (1994). What has become of the psychotherapy of schizophrenia? Acta Psychiatrica Scandinavica Supplementum, 384, 147-152.

Pull, C. (2005). Diagnóstico da esquizofrenia: uma revisão. In M. Maj \& N. Sartorius (Orgs.), Esquizofrenia (pp. 13-70). Porto Alegre: Artmed.

Rosa, M. L. N. M., Silva, R. C. B., Moura-de-Carvalho, F. T., Brandão, M. L., Guimarães, F. S., \& DelBel, E. A. (2005). Routine post-weaning handling of rats prevents isolation rearing-induced déficit in prepulse inhibition. Brazilian Journal of Medical and Biological Research, 38, 1691-1696.

Salgado, J. V., Hetem, L. A., \& Sandner, G. (2006). Modelos experimentais de esquizofrenia - uma revisão. Revista Brasileira Psiquiatria, 28(2), 135-141.

Silva, R. C., Sandner, G., \& Brandão, M. L. (2005). Unilateral electrical stimulation of the inferior colliculus of rats modifies the prepulse modulation of the startle response (PPI): Effects of ketamine and diazepam. Behavior Brain Research, 160(2), 323-330.

Snyder, S. H. (1986). Drugs and the brain (pp. 23-45). New York: Scientific American Library.

Vallada Filho, H., \& Busatto Filho, G. (1996). Esquizofrenia. In P. Almeida, L. Dractu \& R. Laranjeira (Orgs.), Manual de psiquiatria (pp. 127-150). Rio de Janeiro: Guanabara Koogan.

Willner, P. (1984). The validity of animal models of depression. Psychopharmacology, 83(1), $1-16$.

Wyatt, R. J. (2001). Diagnosing schizophrenia. In J. A. Lieberman \& R. M. Murray (Eds.), Comprehensive care of schizophrenia. A textbook of clinical management (pp. 23-34) London: Martins Dunitz.

Zubin, J., \& Spring, B. (1977). Vulnerability - A new view of schizophrenia. Journal of Abnormal Psychology, 86, 103-129.

Recebido em: 20/11/2006

Aceito em: 30/11/2006 